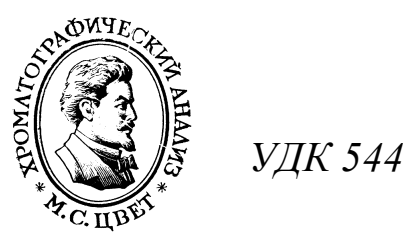

\title{
Сорбенты для ВЭЖХ. Современное состояние и новые направления развития (обзор)
}

\author{
(С) 2021 Яшин А.Я., Яшин Я.И. \\ Группа компаний «Сайтегра», Москва
}

Поступила в редакцию 11.02.2021 г.

DOI: $10.17308 /$ sorpchrom.2021.21/3357

Колонка с сорбентом - основной узел жидкостного хроматографа, так как она выполняет самую важную работу - разделение компонентов смеси. Без разделения сложной смеси невозможно выполнить анализ. Поэтому неслучайно разработке новых сорбентов постоянно уделяется большое внимание. Сорбент обеспечивает постоянное стремление к повышению эффективности колонки, селективности и скорости разделения. Наибольший интерес с момента появления высокоэффективной жидкостной хроматографии (ВЭЖХ) проявляется к сорбентам для обращенно-фазовой хроматографии (ОФХ) - силикагелям с привитыми алкильными группами. Пути их совершенствования - блокировка активных силанольных групп, увеличение диапазона работы с водными элюентами прививкой в алкильную цепь полярных вставок, применение сорбентов с устойчивостью во всем диапазоне $\mathrm{pH}$ (1-14). ОФХ уже 50 лет (1970-2020) лидирует по применению на практике (от 60 до 80\%) в разные годы. Удерживающая способность и селективность сорбентов с привитыми алкильными цепями зависит от числа алкильных цепей или от общего содержания углерода на единицу веса сорбента, которое колеблется от 8 до 20\%. Силикагели со средними размерами пор 80, 100, $120 \AA$ применяют для разделения низкомолекулярных соединений, а силикагели с порами $300 \AA$ - для разделения высокомолекулярных соединений. В обзоре приводятся новые технологи и получения сорбентов: перфузионных, монолитных колонок, сорбентов с порами одного размера, макропористых углеродных адсорбентов. Приведен перечень необычных сорбентов для ВЭЖХ: сверхсшитые полимеры, углеродные нанотрубки, поликапиллярные колонки, жидкие кристаллы, графен и оксид графена, ионные жидкости и др. Приведены перечни сорбентов для хиральной и гидрофильной хроматографии. Сорбенты для хиральной хроматографии на основе полисахаридов, краунэфиров, циклодекстринов, антибиотиков, нанотрубок, наночастиц, ионных жидкостей и др.

В гидрофильной хроматографии применяются сильнополярные сорбенты: гидроксилированный силикагель, модифицированные силикагели с аминопропильными, цианопропильными, диольными, амидными, цвиттерионными группами. Используются в качестве сорбентов в гидрофильной хроматографии ионные жидкости. Процесс совершенствования сорбентов для ВЭЖХ продолжается и нас ждут новые достижения в этом направлении.

Ключевые слова: сорбенты, высокоэффективная жидкостная хроматография (ВЭЖХ), обращенно-фазовый (ОФ) режим, силикагели, разделение, монолитные колонки, сорбенты с порами молекулярных размеров, хиральные сорбенты, структура сорбентов.

\section{Введение}

Колонка, заполненная сорбентом основной узел современного жидкостного хроматографа, так как именно она выполняет главную работу - разделение смеси веществ. Без разделения анализ сложной смеси невозможен. Есть исключение - это применение современного масс-спектрометра высокого разрешения, который в некоторых случаях может проанализировать простые смеси по масс-спектрам. Однако, для анализа сложных смесей компонентов с близки- 
ми свойствами, в частности, изомеров нужно сочетание ВЭЖХ-МС высокого разрешения. Колонка определяет эффективность, селективность и экспрессность разделения. Разделительная способность колонки определяется как эффективностью колонки, так и селективностью сорбента. Пределы детектирования также связаны с работой колонки, чем меньше размывание, тем эффективнее колонка, тем выше амплитуда сигнала.

\section{Краткая история развития сорбентов для ЖХ и ВЭЖХ}

М.С. Цвет предложил хроматографию в виде адсорбционного варианта, он испытал более 100 разных твердых тел в качестве адсорбентов [1]. На первых этапах, в основном, применялся алюмогель, оксид алюминия как адсорбент в жидкостной хроматографии (ЖХ). Затем на смену пришел силикагель, оксид кремния. Эпоха силикагеля продолжается до сих пор. В чистом виде силикагель применяется в нормально-фазовой хроматографии и гель-фильтрационной хроматографии. В обращенно-фазовой хроматографии, в основном, применяются силикагели с привитыми алкильными цепями длиной от $\mathrm{C}_{1}$ до $\mathrm{C}_{30}$, чаще всего применяют сорбенты $\mathrm{C}_{8}$ и $\mathrm{C}_{18}$. Эффективность колонок определяется размером и формой зерен сорбента. В настоящее время используются сферические зерна. Кроме сорбентов с размером 5мкм, стали применять сорбенты с размером 3 и 2 мкм. Силикагели со средними размерами пор 80, 100, $120 \AA$ применяют для разделения низкомолекулярных соединений, а силикагели с порами $300 \AA$ - для разделения высокомолекулярных соединений и биополимеров. Приводим перечень обзоров по сорбентам для ВЭЖХ [2-9]. При применении зерен менее 2 мкм (1.3-1.7 мкм) эффективность колонок возрастает, за счет уменьшения внутренних и внешних путей диффузии увеличивается экспрессность разделения. Однако это потребовало большого входного давления, так возник метод ультраВЭЖХ [10-12] с входным давлением более 1000 атм. Это потребовало разработку новых насосов, новых дозирующих систем и др. частей хроматографа. Это значительно увеличило стоимость оборудования. Поэтому стали думать, как увеличить эффективность колонки без уменьшения размера частиц, чтобы использовать обычную уже разработанную аппаратуру для ВЭЖХ с входным давлением 400-500 атм. Обратили взоры к поверхостнопористым сорбентам (ППС) [13-21], которые имеют высокую эффективность и экспрессность. ППС с размером частиц 3 мкм по эффективности сопоставимо с частицами 1.5 мкм в УВЭЖХ [16], используя недорогую доступную аппаратуру. В ВЭЖХ применяются колонки следующих размеров: обычные аналитические с внутренним диаметром 2.1-4.6 мм, микронабивные с внутренним диаметром 0.5-2.1 мм, капиллярные с диаметром 100-150 мкм и наноколонки с диаметром 20-100 мкм. Предпочтительная длина аналитических колонок составляет $250,150,100,75,50$, и 30 мм.

\section{Основные направления развития сорбентов для обращенно- фазовой хроматографии}

До 1950 г использовался только нормально-фазовый режим жидкостной хроматографии (хотя он так не назывался в то время), который характеризуется тем, что применяют полярный сорбент и неполярные или слабополярные элюенты. В 1950 г [22] был предложен обращенно-фазовый вариант хроматографии (ОФХ). Активные аналитические применения этого режима начаты с 1970 г, когда были разработаны сорбенты для ОФХ - силикагели с привитыми алкильными цепями. Они оказались очень удобными и ОФХ до настоящего времени (т.е. в течение 50 лет) лидирует в аналитических применениях, ее доля в разное время составляла $60-80 \%$ [23]. В ОФХ чаще всего применяется сорбент $\mathrm{C} 18$, тысячи методик разработаны на ос- 
нове колонок с C18. В этом режиме сорбент неизменный, меняется только элюент. Имеет место простая закономерность связи удерживания с концентрацией органической добавки в элюенте. На первом этапе были проведены работы по улучшению сорбентов для ОФХ. Прежде всего, улучшили основусиликагель, разработали технологию получения частиц в виде сфер размером 5 мкм, очистили от примесей металлов. В настоящее время силикагель содержит менее 3-5 ppm примесей металлов, что практически исключает вклады каталитических процессов (Purospher star RP18). В таблице 1 приведены основные направления развития сорбентов для ОФХ. Основной недостаток ОФХ вклад в удерживание остаточных силанольных групп. Из-за пространственных затруднений половина этих групп остаются свободными и они стали доступны для небольших молекул, что приводит к несимметричным пикам и нарушению закономерности удержива- ния. Предприняты усилия для блокировки силанольных групп, используя разные процессы endcapping, в частности, прививкой сразу двух связанных алкильных групп (бидентатных) или алкильных цепей с изопропильными или изобутильными заместителями [30, 31]. Следующий недостаток ОФХ сорбентов - это схлопывание алкильных цепей при больших содержаниях воды в элюенте, наступает и коллапс в удерживании соединений. Для исключения этого эффекта стали включать в середину алкильных цепей полярные вставки разной природы использовали процесс embedding [33-35], что увеличило селективность к полярным соединениям и позволило работать с 100\% водными элюентами [44-46]. Для увеличения селективности разделения некоторых классов соединений стали использовать сорбенты с привитыми к концу алкильных цепей функциональных групп $\mathrm{CN}, \mathrm{NH}_{2}$, диольные, фенильные и дифенильные группы [36, 37]. C другой стороны, для увеличения гидро-

Таблица 1. Основные направления развития сорбентов для обращенно-фазовой хроматографии

Table 1. The main developmental directions of sorbents for reversed phase chromatography

\begin{tabular}{|c|c|c|c|}
\hline № & Новые направления & Сущность предложений & Ссылки \\
\hline 1 & $\begin{array}{c}\text { Предложена прививка алкильных } \\
\text { цепей к поверхности силикагеля } \\
\text { (щеточные сорбенты) }\end{array}$ & Связь Si-O-C неустойчива & 24 \\
\hline 2 & Предложена более стабильная связь & -Si-O-Si- & 25 \\
\hline 3 & $\begin{array}{c}\text { Предложена очистка исходного си- } \\
\text { ликагеля от примесей металлов }\end{array}$ & $\begin{array}{c}\text { Уменьшение адсорбционной и } \\
\text { каталитической активности }\end{array}$ & 26 \\
\hline 4 & $\begin{array}{c}\text { Уменьшение вклада в удерживание } \\
\text { силанольных групп }\end{array}$ & $\begin{array}{l}\text { Блокировка этих групп } \\
\text { (endcapping) }\end{array}$ & $27-32$ \\
\hline 5 & $\begin{array}{c}\text { Алкильные цепи при большом со- } \\
\text { держании воды в элюенте схлопы- } \\
\text { ваются, наступает коллапс }\end{array}$ & $\begin{array}{c}\text { Вставка в алкильную цепь по- } \\
\text { лярных групп (процесс embed- } \\
\text { ded) }\end{array}$ & $33-35$ \\
\hline 6 & $\begin{array}{c}\text { Увеличение полярности ОФХ } \\
\text { сорбентов }\end{array}$ & $\begin{array}{c}\text { Прививка на конце алкильных } \\
\text { цепей групп } \mathrm{CN}, \mathrm{NH}_{2} \text {, диольных, } \\
\text { фенильных, дифенильных }\end{array}$ & 36,37 \\
\hline 7 & $\begin{array}{c}\text { Увеличение гидрофобности сорбен- } \\
\text { тов для ОФХ }\end{array}$ & $\begin{array}{c}\text { Перфторирование алкильных } \\
\text { цепей }\end{array}$ & 38,39 \\
\hline 8 & $\begin{array}{c}\text { Увеличение стабильности колонки } \\
\text { во всем диапазоне рН элюента }\end{array}$ & $\begin{array}{c}\text { Применение устойчивых сор- } \\
\text { бентов к рН (1-14) } \\
\text { - оксид титана } \\
\text { - оксид циркония } \\
\text { - углеродные адсорбенты } \\
\text { - полимерные адсорбенты }\end{array}$ & $\begin{array}{l}40 \\
41 \\
42 \\
43\end{array}$ \\
\hline
\end{tabular}


фобности сорбентов стали применять перфторированные алкильные цепи [38, 39]. Существенный недостаток ОФХ сорбентов на основе - неустойчивость к $\mathrm{pH}$ элюента, они работают устойчиво в диапазоне $\mathrm{pH}$ 1.5-8. Были предложены диоксиды, стабильные во всем диапазоне $\mathrm{pH}$ (1-14), это оксиды титана [40] и циркония [41], затем стали применять гидрофобные макропористые углеродные [42] и полимерные [43] сорбенты, которые могут также работать во всем диапазоне $\mathrm{pH}$.

\section{Динамическое модифицирование сорбентов в обращенно-фазовой хроматографии (ОФХ)}

В классической ОФХ сильнополярные соединения слабо удерживаются и плохо разделяются, для этих целей был предложен метод динамического модифицирования поверхности полярными группами за счет их добавки в элюент [47, 48]. Происходит насыщение поверхности полярными группами с образованием поверхностного слоя, на нем сорбируются полярные соединения, повышается удерживание и улучшение разделения в варианте ион-парной хроматографии [7-9]. Если убрать из элюента полярную добавку, то через определенное время она полностью вымывается и сорбент возвращается к первоначальному состоянию. Таким образом, было показано, что на поверхности можно создать слой хиральных молекул и разделять оптические изомеры, т.е. реализовать метод хиральной хроматографии на основе сорбентов ОФХ [49]. Это весьма удобно и дешево. Динамическое модифицирование алкильных цепей в ОФХ может быть универсальным методом, расши- ряющим аналитические возможности этого метода. Селективность определяется химической природой поверхности сорбентов. Удерживающая способность и селективность зависит от числа привитых алкильных цепей или от общего содержания углерода на единицу веса сорбента, которая колеблется от 8 до 20\% [50]. Кроме привитых алкильных цепей стали прививать цепи с полярными концевыми функциональными группами [7-9, 51]. Следует еще выделить важную связь удерживания со структурой молекул [52], влияние на удерживание энтальпии и энтропии сорбции [54], роль температуры в ВЭЖХ, влияние геометрической структуры сорбентов с удерживанием и селективностью [56].

Далее в отдельных разделах будут описаны новые технологии получения сорбентов и приведен перечень необычных применяемых сорбентов.

\section{Новые технологии создания сорбентов для ВЭЖХ}

Перфузионная хроматография (табл. 2). Перфузионная хроматография (ПХ) впервые предложена в 1990 г [57], позднее разработана ее теория [58]. Перфузионные колонки занимают промежуточное положение между обычными насадочными и монолитными колонками. Этот метод чаще используется для препаративного выделения веществ (в частности, белков) в чистом виде и реже для аналитических целей $[59,60]$. В перфузионных колонках микропотоки элюента проходят сквозь зерна сорбента, а не огибают их, как в обычных насадочных колонках, поэтому нет вклада вихревой диффузии в общее размывание. В качестве сорбентов в ПХ исполь-

Таблица 2. Новые технологии создания сорбентов для ВЭЖХ

Table 2. New technologies for creating sorbents for HPLC

\begin{tabular}{|c|c|c|}
\hline № & Новые технологии & Ссылки \\
\hline 1 & Перфузионные сорбенты & $57-60$ \\
\hline 2 & Монолитные колонки & $61-66$ \\
\hline 3 & Сорбенты с порами молекулярных размеров & $74-80$ \\
\hline 4 & Технология получения макропористых углеродных адсорбентов & $96-103$ \\
\hline
\end{tabular}


зуют сополимеры стирола и дивинилбензола. Такие сорбенты представляют собой частицы диаметром 20 мкм, содержащие два типа пор: большие поры (6000-8000 А), по которым элюент проходит через зерна, и диффузионные поры (800-1500 А), по которым молекулы соединений проникают в поры сорбента. Из-за ускорения процесса массообмена в перфузионных колонках можно использовать большие скорости элюента, сокращая время разделения. Перфузионные сорбенты производят для разных вариантов хроматографии: ОФХ, нормально-фазовой, ионообменной, гидрофобной и аффинной.

Монолитные колонки. Впервые монолитные колонки предложены в работе [61]. Монолитные колонки на основе силикагеля промышленно изготовлены фирмой «Мерк» под названием «Хромолит» [62]. В монолитных колонках сорбционный слой синтезируется непосредственно в колонках в виде непрерывного стержня. В сорбционном слое создаются крупные транспортные поры и мезопоры для сорбции молекул разделяемых соединений. Основное преимущество монолитных колонок - это небольшое входное давление и в связи с этим малое сопротивление потоку [63-66]. Это позволяет соединять колонки последовательно и использовать для анализа колонки большой длины, что недоступно для насадочных колонок [67]. Обычно в ВЭЖХ применяют колонки длиной не более 25 см с общей эффективностью 25000 теоретических тарелок (тыс.т.т.). Средняя эффективность колонок в ВЭЖХ около 100 тыс.т.т. на метр длины колонки. В большинстве случаев в распоряжении аналитиков в ВЭЖХ колонки с общей эффективностью 10-25 тыс.т.т. Испытана монолитная колонка общей длиной 140 см, составленная из 14 последовательно соединенных колонок длиной 10x4.6 мм. Достигнута общая эффективность 108 тыс.т.т. (по алкилбензолам). При этом входное давление было всего 117 бар [67]. В 2012 г были предложены монолитные колонки второго поколения «Хромолит ВР» с более высокой эффективностью (140 тыс.т.т./м) [68]. Эффективность монолитных колонок первого поколения 80 тыс. т.т.. Монолитные колонки создаются и на полимерной основе [69, 70]. В качестве полимерных материалов были использованы: стирол с дивинилбензолом, полимеры на основе акрилатов, метакрилатов, глицидилметакрилата и др. Получены монолитные колонки разного диаметра: аналитические, капиллярные микронасадочные, препаративные и даже наноколонки для ЧИПов [71]. Кроме универсальных ОФ монолитных колонок, разработаны специализированные колонки для разделения энантиомеров [72], белков, полинуклеотидов [73]. Монолитные колонки применяют в многомерных вариантах хроматографии [73].

Сорбенты с порами молекулярных размеров (molecularly imprinted polymers) (табл. 2). Сорбенты этого типа суперселективные, т.к. они могут сорбировать молекулы только одного размера или близких групп молекул [74]. Синтез таких сорбентов описан в работах [75-80]. Они синтезируются на полимерной основе, в частности, на основе метакриловой кислоты и этилендиметакрилата. В первую очередь эти сорбенты применяют для селективной экстракции и концентрирования, реже для разделения. Для их обозначения используют выражение «молекулярное распознавание» (molecular recognition). Сорбенты с порами молекулярных размеров применяют для селективного выделения супертоксикантов 3,4-бензпирена, хлордиоксинов, пестицидов в окружающей среде [81-83], нитрозаминов и афлатоксинов в пище [84, 85]. В пищевых продуктах определяют полезные соединения: кверцетин [86], антоцианины [87], кофеин [88], катехины [89], в биологических жидкостях аминокислоты, пептиды, белки [90], вирусы [91], клетки [92], нуклеозиды, холестерин [93]. Такие сорбенты применяют для разделения энантиоме- 
ров [94], для доставки лекарств к нужным органам [95].

Получение однородных макропористых углеродных адсорбентов. В 1979 г в работах [96] был предложен способ получения макропористых углеродных адсорбентов с однородной поверхностью как у графитированной термической сажи [97] по технологии шаблонов (template). В качестве шаблонов были использованы макропористые силикагели со сферическими частицами 5 мкм. Поры таких силикагелей заполняются фенил-формальдегидным полимером. Затем он карбонизируется нагревом в инертной среде до $1000^{\circ} \mathrm{C}$, при этом в порах образуются углеродные частицы. Силикагелевый каркас растворяют в 5 М растворе $\mathrm{NaOH}$ и остается макропористый углеродный адсорбент, т.е. зеркальная реплика силикагеля. На этой стадии поверхность углеродного адсорбента $400 \mathrm{~m}^{2} / \Gamma$ с порами $30 \AA$. Графитизация при температуре $2000^{\circ} \mathrm{C}$ приводит к перестройке структуры однородного адсорбента, удаление микропор и создание однородной кристаллической поверхности. После графитизации остаются поры, которые были в исходном силикагеле. Впервые серийное производство таких макропористых углеродных адсорбентов освоила фирма «Шендон» под названием Hypercarb. Структурные характеристики их следующие: средний диаметр пор $250 \AA$, удельная поверхность $120 \mathrm{~m}^{2} / \Gamma$, объем пор 75\%, размер частиц 5 или 7 мкм. Они стабильны во всем интервале рН (1-14), не растворяются, не набухают ни в одном растворителе, выдерживают давление до 400 атм.

Таблица 3. Необычные сорбенты для ВЭЖХ Table 3. Unusual HPLC sorbents

\begin{tabular}{|l|l|l|}
\hline$№$ & Название сорбентов для ВЭЖХ & Ссылки \\
\hline 1 & Сверхсшитые полимерные сорбенты & 104 \\
\hline 2 & Углеродные нанотрубки & 105 \\
\hline 3 & Графен, оксид графена & 106,107 \\
\hline 4 & Ионные жидкости & 108 \\
\hline 5 & Поликапиллярные колонки & 109,110 \\
\hline 6 & Жидкие кристаллы & 111,112 \\
\hline 7 & Титановые монолитные колонки & 113 \\
\hline
\end{tabular}

Колонки, наполненные hypercarb имеют эффективность 60 тысяч т.т. на метр длины колонки. Эти адсорбенты нашли применение для разделения ароматических соединений, структурных изомеров, разных фармпрепаратов, смесей катионов и анионов [98]. В ОФХ они показывают необычную закономерность удерживания [99], в частности, удерживание полярных фенольных соединений. Колонки с макропористыми углеродными адсорбентами можно использовать для разделения карбогидратов [100]. Аналитические возможности колонок с hypercarb описаны в работах [101-103].

Необычные сорбенты для ВЭЖХ (табл. 3). На сверхсшитых полимерных сорбентах закономерности удерживания промежуточные между нормальнофазовым и обращенно-фазовым режимами [104]. Порядок удерживания на углеродных нанотрубках [105] и графене [106] и оксиде графена [107] также отличается от порядка удерживания в ОФХ. Ионные жидкости придают сильную полярность сорбентам [108]. Поликапиллярные колонки, в основном, используются в газовой хроматографии. Однако, в работах $[111,110]$ показано, что они могут успешно использоваться и в ВЭЖХ для экспрессных разделений. На жидких кристаллах хорошо разделяются изомеры [111, 112]. На титановых монолитных колонках можно работать во всем диапазоне рН (1-14) [113].

Сорбенты для хиральной хроматографии. В последние годы хиральная хроматография получила широкое развитие, особенно для разделения энантиомеров лекарств [105]. Хиральная хроматогра- 
фия в ВЭЖХ предложена В.А. Даванковым в варианте лигандообменной [114]. К настоящему времени разработаны сотни сорбентов для хиральной хроматографии, в которых активным центром является ассиметричные центры (три заместителя разной природы). Основные сорбенты для хиральной хроматографии созданы на основе полисахаридов [107], краунэфиров [108], циклодекстринов [109], антибиотиков [110], нанотрубок [111], наночастиц [112], ионных жидкостей [113] и многих других [114].

Сорбенты для гидрофильной хроматографии. В гидрофильной хроматографии используются полярные сорбенты и полярные элюенты. Она очень удобна для быстрого разделения сильнополярных соединений $[123,124]$. Для этих целей применяются чистые гидроксилированные силикагели, аминированные и нитрильные сорбенты [125-128] и др.

Тесты для сравнения сорбентов в ОФХ. Танака предложил тест для оценки сорбентов в ОФХ.

1. Оценка емкости сорбентов по пентилбензолу.

2. Оценка селективности к метиленовой группе $\left(\mathrm{CH}_{2}-\right)$ по определению отношения пентилбензола к бутилбензолу.

3. Оценка стерической селективности по отношению удерживания трифенилена к о-терфинилу.

4. Оценка силанольной активности по отношению удерживания кофеина к фенолу.

5. Оценка ионообменной емкости при pH 7.6 по отношению удерживания бензиламина к фенолу.
6. Оценка ионообменной емкости при pH 2.7 по отношению удерживания бензиламина к фенолу.

Таким путем в этом тесте производится всесторонняя оценка сорбентов при их выборе. Эльгельгардт предложил свой тест с использованием десятка соединений.

Выбор сорбентов. К настоящему времени разными фирмами разработано более 1000 колонок для ВЭЖХ и каждый год появляется 50 новых. Для быстрого выбора нужной колонки создана база данных ACD (Labs column selection database. ACD column selection), в которой приведены их характеристики по системе Танака [129].

\section{Заключение}

В обзоре отмечена огромная роль колонок, заполненных сорбентами в ВЭЖХ. Показано большое разнообразие сорбентов для разных вариантов ВЭЖХ, разработка новых сорбентов всегда была актуальной темой. Предложено много новых технологий создания уникальных сорбентов с новыми аналитическими возможностями.

От сорбентов зависят самые важные характеристики: удерживание, селективность разделения, эффективность колонок. Экспрессность разделения также определяется, в основном, сорбентом.

Процессы создания новых сорбентов и совершенствование известных продолжаются, это способствует прогрессу в ВЭЖХ. 


\title{
Sorbents for HPLC. \\ Current state and new directions of development (Overview)
}

\author{
(C) 2021 Yashin A.Ya., Yashin Ya.I. \\ Scietegra Group of Companies, Moscow, Russian Federation
}

\begin{abstract}
A column with a sorbent is the main unit of a liquid chromatograph, since it performs the most important operation - the separation of a mixture's components. Analysis cannot be performed without the separation of a complex mixture. Therefore, attention is constantly paid to the development of new sorbents. The sorbent provides a constant drive to the improvement of column efficiency, selectivity, and separation speed. Since the advent of high performance liquid chromatography (HPLC), the greatest interest has been shown to sorbents for reversed phase chromatography (RPC) - silica gels with embedded alkyl groups. The methods of their improvement are endcapping, increasing the range of operation with aqueous eluents by embedding of polar groups into the alkyl chain, and using sorbents with stability over the entire $\mathrm{pH}$ range (1-14). For 50 years (1970-2020) RPC has been the leader in practical applications (from 60 to $80 \%$ ) in various years. The retention capacity and selectivity of sorbents with embedded alkyl chains depends on the number of alkyl chains or on the total carbon content per unit weight of the sorbent, which ranges from 8 to $20 \%$. Silica gels with an average pore size of $80,100,120 \AA$ are used to separate low molecular weight compounds, and silica gels with pores of $300 \AA$ are used to separate high molecular weight compounds. The review presents new technologies for the production of sorbents: perfusion, monolithic columns, sorbents with pores of the same size, and macroporous carbon adsorbents. A list of unusual sorbents for HPLC is given: hypercross-linked polymers, carbon nanotubes, polycapillary columns, liquid crystals, graphene and graphene oxide, ionic liquids, etc. Lists of sorbents for chiral and hydrophilic chromatography are presented.

Sorbents for chiral chromatography are based on polysaccharides, crown ethers, cyclodextrins, antibiotics, nanotubes, nanoparticles, ionic liquids, etc. In hydrophilic chromatography, strongly polar sorbents are used: hydroxylated silica gel, modified silica gels with aminopropyl, cyanopropyl, diol, amide, and zwitterionic groups. Ionic liquids are used as sorbents in hydrophilic chromatography. The process of improving sorbents for HPLC continues and new achievements in this direction await us.
\end{abstract}

Keywords: sorbents, high performance liquid chromatography (HPLC), reverse phase (RP) mode, silica gels, separation, monolithic columns, molecularly imprinted polymers, chiral sorbents, sorbent structure.

\section{Список литературы/References}

1. Tsvet M.S. Khromofilly v rastitel'nom i zhivotnom mire. Varshava. Izd-vo Varshavskogo universiteta, $1910,380 \mathrm{p}$.

2. Gritti F., Guiochon G., J. Chromat. A, 2012, Vol. 1228, pp. 2-19.

3. Qia H., Liang Y., Sun M., Jung S., Anal. Bioanal. Chem,. 2011, Vol. 399, pp. 3307-3322.

4. Unger K.K., Lamotte S., Machtejevas S., Liquid Chromatography, 2017, pp 39-89.

5. Grinias J.P., Godinho J.M., Gritti F. et al., LC GC North. Am., 2020, No 6, pp. 75-81.

6. Ghamat S.N., Tolebpour Z., Mehdi A., Trends Anal. Chem., 2019, Vol. 118, pp. 556-573.

7. Zuvela R., Skoczylas M., Lin J.J., Baczek T. et al., Chem.Rev., 2019, Vol. 119, pp 3674-3729.

8. Unger K.K., Liapis A.I., J. Sep. Sci., 2012, Vol. 95, pp 1201-1210.

9. Hannai T., Separations, 2020, Vol. 6, pp. 2-19. 10.Walter T.H., Andrews R.W., Trends Anal Chem., 2014, Vol. 63, pp 14-20.
11. De Vos J., Broeckhoven K., Eeltink S., Anal. Chem., 2016, Vol. 88, pp. 262-278.

12.Yashin Ya.I., Vedenin A.N., Yashin A.Ya., Analitika, 2015, No 2, pp. 432-1434.

13.Kirkland J.J., Langlois T., DeStefano J., Am. Lab., 2007, Vol. 39, pp. 18-21.

14.Fekete S., Fekete J., Ganzler K., J. pharm. Biomed. Anal., 2009, Vol. 49, pp. 64-71.

15. Guiochon G., Gritti F., J Chrom. A, 2011, Vol. 1218, pp. 1915-1938.

16.McCalley D.V., J. Chrom A, 2011, Vol. 1218, pp. 2887-2897.

17. McCalley D.V., J. Chrom. A, 2008, Vol. 1193, pp. 85-101.

18. Horvath C.G., Preiss B.A., Lipsky S.R., Anal. Chem., 1967, Vol. 39, pp. 1422-1428.

19. Zhdanov S.P., Kiselev A.V., YAshin YA.I., Zh.fiz. khimii, 1963, Vol. 37, pp. 1432-1434.

20. Yaschin Ya.I., Zhdanov S.P., Kiselev A.V., Gas Chromatografie, 1963, Leuna, pp. 402-415. 
21. Poole C.F., Atapaltu C.N., J.Chrom. A, 2020, Vol. 1633, pp. 461652

22. Howard G.A., Martin A.J.P., Biochem. J., 1950, Vol. 46, pp. 532-538.

23. Brandt A., Mann G., Arlt W., J. Chrom. A, 1998, Vol. 796, pp. 223-228.

24. Halasz I., Sebastian I., Angew. Chem. Inter., 1969, Vol. 8, pp 453-454.

25. Kirkland J.J., DeStefano J.J., J.Chromat. Sci., 1970, Vol. 8, pp. 309-315.

26. Purospher Star RP 18 Sigma-Aldrich

27. Meyer E., Engelhardt H., Fresenius Z. Anal Chem., 1989, Vol. 333, pp. 734-737.

28. Bocian S., Buszewski B., J.Sep.Sci., 2012, Vol. 35, pp. 1191-1200.

29. Kiridena W., Poole C.F., Koziol W.W., Chromatographia, 2003, Vol. 57, pp. 703-707.

30. Kirkland J.J., Adams J.B., van Straten M.A., Claessens H.A., Anal Chem., 1998, Vol. 70, pp. 4344-4348.

31. Yashin Ya.I., Yashin A.Ya., Ros. Khim. Zhurn., 2003, Vol. 47, pp. 64-79.

32. Yashin Ya.I., Yashin A.Ya., Uspekhi khimii, 2006, Vol. 35, pp. 366-379.

33. Rommer C.A., Sander L.C., Anal.Bioanal. Chem., 2009, Vol. 394, pp. 285-291.

34. O Sullivan G.P., Scully N.M., Glennan J.D., Anal. Lett., 2010, Vol. 43, pp. 1609-1629.

35. Wilson N.S., Gilroy J., Dolan J.W., Snyder L.R., J. Chrom. A, 2004, Vol. 1026, pp. 91-100.

36.Lopez D.A., Green A.I., Bell D.S., $L C G C$ Europe, 2020, Vol. 33, pp. 460-465.

37. Neue U.D. HPLC column. Theory, technology and practice, NY Wiley-VCH, $1997,395 \mathrm{p}$.

38. Zhang W., J.Fluorine Chem., 2008, Vol. 129, pp. 910-919.

39. Przybyciel M., LC GC Europe, 2006, Vol. 19, pp. 19-27.

40. Jiang Z.T., Zuo Y.M., Se $P u, 2001$, Vol. 19, pp. 297-300.

41. Dunlop C., McNeff, Stoll D., Carr P., Anal.Chem., 2001, Vol. 73, pp. 598A-607A.

42. Leport C., Gunatillaka A.D., Poole C.F., Analyst, 2001, Vol. 126, pp. 1-9.

43. Gawdzik B., Osypiuk J., J. Chrom. A, 2000, Vol. 898, pp. 13-21.

44. Majors R.E., Przybycill M., LC GC North America, 2002, Vol. 20, pp. 584-588.

45. Nagal N., Enami T., Doshi S., LC GC North America, 2002, Vol. 2, pp. 964-972.

46. Dembek M., Bocian S., Trends Anal. Chem., 2020, Vol. 123, pp. 1-12.
47. Kabulov B.D., Zalyalieva S., Ruzimuradov O.N. et al., Rus. J. Phys. Chem., 2002, Vol. 76, pp. 1512-1514.

48. Brykina G.D., Zharikova V.S., Matusova S.M., Shpigun O.A., J. Anal Chem., 2005, Vol. 60, pp. 1041-1045.

49. Davankov V.A., Bochkov A.S., Kurganov A.A., Roumeliotis P. et al., Chromatographia, 1980, Vol. 13, pp. 677-685.

50. Bereznitski Y., Jaroniec M., Gangoda M.E., J. Chrom.A, 1998, Vol. 828, pp. 59-73.

51. Sander L.C., Wise S.A., Anal. Chem., 1984, Vol. 56, pp. 504-510.

52. Kaliszan R., Chem. Rev., 2007, Vol. 107, pp. 3212-3246.

53. Ranatunga R.R.J., Carr P.W., Anal Chem., 2000, Vol. 72, pp. 5679-5692.

54. Guillarme D., Heinisch S., Rocca J.L., $J$. Chrom. A, 2004, Vol. 1052, pp. 39-51.

55. Teutenberg T., Hollebekkers K., Wiese S., Boergers A., J. Sep.Sci., 2009, Vol. 32, pp. 1262-1274.

56. Vailaya A., Horvath C., J.Chrom. A, 1998, Vol. 829, pp. 1-27.

57.Afeyn N.B., Fulton S.P., Gordon N.F. et al., J. Chrom. A, 1990, Vol. 519, pp. 233-266.

58.Liapis A.T., McCoy M.A., J. Chrom A, 1992, Vol. 599, pp. 87-95.

59.Lloyd L.L., Warner F.P., J. Chrom A, 1990, Vol. 512, pp. 365-376.

60.Hjerten S., Liao J.L., Zhang R., J. Chrom $A, 1989$, Vol. 473, pp. 273-275.

61.Hjerteu S ., Liao J.L., Zhang R., J. Chromat, 1989, Vol. 473, pp. 273-278.

62.Unger K.K., Skudas R., Shulte M.M., J. Chrom. A, 2002, Vol. 1184, pp. 793-815.

63.Guiochon G., J. Chrom A, 2007, Vol. 1168, pp. 101-168.

64.Tanaka N., Kobayashi H., Nakanishi K. et al., Anal.Chem., 2001, Vol. 73, pp. 420-430.

65.Jandera P., Hajek T., Sromova Z., J. Sep.Sci., 2019, Vol. 42, pp. 670-677.

66.Hormann K., Mullner T., Bruns S. et al., $J$. Chrom A, 2012, Vol. 1212, pp. 48-58.

67.Miyamoto K., Hara K., Kobayashi H. et al., Anal. Chem., 2008, Vol. 80, pp. 8741-8750.

68. Jandera P., J. Chrom. A, 2013, Vol. 1313, pp 37-53.

69. Sotnikova Yu.S., Patrushev Yu.V., Vestnik Tomsk. Gos. Un-ta, khimiya, 2019, Vol. 14, pp. 40-52.

70. Patruchev Y.V., Yudina Y., Sidelnikov V., J. Liq.Chrom, 2018, Vol. 41, pp. 458-466.

71. Rieux L., Sneekes E.J., Swart R., LCGC North America, 2011, Vol. 29, Is. 10, pp. 926-934. 
72. Josic D et al., J. Chrom. A, 2001, Vol. 752, pp 101-105.

73. Tanaka N., Kimura H., Takuda D. et al., Anal. Chem., 2004, Vol. 46, pp. 1278-1281.

74.Wulff G., Sarhan A., Angew. Chem. Int. Ed. Engl., 1972, Vol. 11, pp. 341-345.

75.Wulff G., Sarhan A., Zabrocki K., Tetrahedron Letters, 1973, Vol. 14, Issue 44, pp. 4329-4332.

76.BelBruno J.J., Chem. Rev., 2019, vol. 119, pp. 94-119.

77.Chen L., Wang X., Lu W., Wu X. et al., Chem. Soc. Rev., 2016, Vol. 45, pp. 2137-2211.

78.Wei Z.-H., Mu L.N., Huang Y.P., Lin Z.S., Trends Anal. Chem., 2017, Vol. 86, pp. 84-92.

79. Handbook of molecularly imprinted polymers. Eds. C.Alvarez-Lorenzo, A.Concheira. Smithers Rapra Technology Ltd. Shropshire UK 2013. 400 p.

80. Molecular imprinting Ed. K.Haupt. Springer. Berlin Germany 2012, 265 p.

81. Lai J.P., Niessner R., Knopp D., Anal. Chim. Acta, 2004, Vol. 522, pp. 137-144.

82. Ndunda E.N., Mizaikoff B., Analyst, 2016, Vol. 141, pp. 3141-3156.

83.Bjarnason B., Chimuka L., Ramstrom O., Anal.Chem., 1999, Vol. 71, pp. 2152-2156.

84.Xiaoman Zheng, Tongguang Xu, Rui Shi, Nan Lu, et al., Materials Letters, 2018, Vol. 211, pp. 21-23.

85.Song L, He J, Chen N, Huang Z., J Sep Sci., 2019, Vol. 42, No 24, pp. 3679-3687.

86.Molinelli A., Weiss R., Mizaikoff B., J. Agric. Food Chem., 2002, Vol. 50, pp. 1804-1808.

87.Zhao Q.Y., Zhao H.T., Yang X. et al.,

J.Chrom.A, 2018, Vol. 1572, pp. 9-19.

88.Wang D., Hong S.P., Row K.H., Kor. J. Chem. Eng., 2004, Vol. 21, pp. 853-857.

89.Ma N., Row K.N., Molecules, 2020, Vol. 25, pp. 280-295.

90.Kempe M., Mosbach K., J. Chromatogr. A, 1995, Vol. 691, pp. 317-323.

91.Gast M., Sobek H., Mizaikoff B., $\operatorname{Tr} A C$ Trends in Analytical Chemistry, 2019, Vol. 114, pp. 218-232.

92.Piletsky S., Canfarotta F., Poma A. et al., Trends in biotechnology, 2020, Vol. 38, pp 368-387.

93. Sellergren B., Wieschemeyer J., Boos K., Seidel

D., Chem. Mater., 1998, Vol. 10, pp. 4037-4046.

94.Sellergren B., J. Chromatogr. A, 2001, Vol. 906, pp. 227-252.

95. Alvarez-Lorenzo C., Concheiro A., J. Chromatogr. B, 2004, Vol. 804, pp. 231-245.

96.Knox P., Ross P., Adv. Chromat., 1998, Vol. 37, pp. 73-95.

97.Forgacs E., Cserhati T., Trends Anal.
Chem., 1995, Vol. 14, pp 23-29.

98.Pereira L., J. Liq. Chrom Rel.Techn., 2008,

Vol. 31, pp. 1687-1731.

99.Czerhati T., Biomed Chromat., 2009, Vol. 23, pp. 111-118.

100. Yashkin S.N., Solovova N.V., ZH.fiz. khimii, 2004, Vol. 78, pp. 344-347.

101. Enerly M.R., LC GC Europe, 2000, Vol. 103, pp. 685-694.

102. Kartsova L.A., Makarov A.A., ZHurn. priklad. Khimii, 2002, Vol. 75, No 11, pp.1761-1767.

103. Shcherbakova K.D., YAshin YA.I., V knige «100 let khro-matografii»» Otv. Red. B.A.Rudenko, M., Nauka, 2003, pp. 670-697.

104. Davankov V., Tsyurupa M., Ilyin M., Pavlova L., J. Chromat. A, 2002, Vol. 965, pp. 65-73.

105. Chang Y.X., Zhou L.L., Li G.X. et al., J. Liq. Cromat., 2007, Vol. 30, pp. 2953-2956.

106. Yang L., Guo Xiao T., Yang J. et al., J. Chrom. Sep. Techn., 2018, Vol. 8, pp. 399-405.

107. Czajek H., Jonic J., Wawer T., Purchola M., Crit. Rev. Anal.Chem., 2020, Vol. 50, pp. 445-471.

108. Shi X., Qiao L., Xu G., J. Chrom. A, 2015, Vol. 1420, pp. 1-15.

109. Zhdanov A.A., aftoreferat, Novosibirskij Gosudarstvennyj universitet, 2011, 24 p.

110. Sidel'nikov V.N., Analitika, 2014, No 6, pp. 40-55.

111. Aratskova A.A., Vetrova Z.P., Yashin Ya.I., J. Chrom. A, 1986, Vol. 365, pp. 27-30.

112. Sargazi M., Linford M.R., Kaynhaii M., Crit. Rev. Anal. Chem., 2019, Vol. 19, pp. 243-255.

113. Randon J., Gullrin J.F., Rocca J.L., $J$. Chrom A, 2008, Vol. 1214, pp. 183-186.

114. Davankov V.A., Bochkov A.S., Kurganov A.A. et al., Chromatographia, 1980, Vol. 13, pp. 677-685.

115. Ikai T, Okamoto Y., Chemical Reviews, 2009, Vol. 109, No 11, pp. 6077-6101.

116. Adhikari S., Lee W., J. Pharm. Investig., 2018, Vol. 48, pp. 225-231.

117. Mitchell C.R., Armstrong D.W., Cyclodextrin-Based Chiral Stationary Phases for Liquid Chromatography. In Chiral Separations: Methods and Protocols; Gübitz, G., Schmid, M.G., Eds.; Humana Press: New York, NY, USA, 2004, pp. 61-112.

118. Ward T.J., Farris A.B., J Chromatogr A, 2001, Vol. 906, No 1-2, pp. 73-89.

119. Ahmed M., Yajadda M.M.A., Han Z.J., Su D. et al., J. Chromatogr. A, 2014, Vol. 1360, pp. 100-109.

120. Shukla N., Bartel M.A., Gellman A.J., 
J. Am. Chem. Soc., 2010, Vol. 132, pp. 8575-8580.

121. Fernandes C., Tiritan M., Pinto M., Symmetry, 2017, Vol. 9, pp. 206-209.

122. Tu F.Y., Yu L.Y., Yu J.G., Chen X.Q. et al., Nano, 2013, Vol. 8, 1350069.

123. Jandera P., Janas P., Anal. Chim. Acta, 2017, Vol. 967, pp. 12-32.

124. Ponten J.M., LC GC North Am, 2012, Vol. 30, pp 26-42.

125. Olsen B.A., J. Chrom A, 2001, Vol. 913, pp. 113-122.

Яшин Яков Иванович - д.х.н., профессор, научный консультант группы компаний «Сайтегра», Москва

Яшин Александр Яковлевич - к.х.н., старший научный сотрудник $\mathrm{OOO}$ «Институт аналитической токсикологии», Москва
126. Jandera P., Anal. Chim. Acta, 2011, Vol. 1692, pp 1-25.

127. Osipov A.S., Popova O.A., Gretskaya T.H. et al., The Bulletin of the Scientific Centre for Expert Evaluation of Medicinal Products, 2018, Vol. 8, No 3, pp. 162-169

128. McCalley, J. Chrom. A, 2008, Vol, 1193, pp. 85-91.

129. Kinata K., Iwaguchi K., Ohishi S., Jinno K., Eksteen R. et al., J. Chrom. Sci., 1989, Vol. 27, pp 721-728.

Yashin Yakov I. - Dr.Sci. (chemistry) professor, Scietegra Group of Companies, Moscow

Yashin Alexander Ya. - Dr.Sci. (chemistry), Senior Researcher, Institute of Analytical Toxicology LLC, Moscow 